\title{
THERMAL STRESSES ANALYSIS IN AN ISOTROPIC MATERIAL DISC SUBJECTED TO THERMAL LOAD AND DENSITY WITH RIGID INCLUSION
}

\author{
Pankaj Pankaj ${ }^{1}$ and Naresh Kumar ${ }^{1}$ \\ ${ }^{1}$ ICFAI University Solan
}

October 14, 2020

\begin{abstract}
The objective of this paper is to present the study thermal stresses in an isotropic material disc with rigid inclusion and subjected to mechanical load and density parameter by using transition theory. The transition theory includes classical macroscopic solving problems in plasticity, creep relaxation, and semi-empirical yield conditions. It has been observed that the radial stress has a maximum value at the internal surface of the disc made of compressible material (i.e. copper) as compared to the disc made of incompressible material (i.e. rubber). With the introduction of thermal condition, density parameter and load, the values of radial and circumferential stress increase of the internal surface of compressible/ incompressible materials. The displacement component increased at the outer surface of the disc made of compressible /incompressible materials and fully plastic stage. Results have been discussed numerical and depicted graphically.
\end{abstract}

\section{Hosted file}

mma final.pdf available at https://authorea.com/users/367023/articles/486615-thermalstresses-analysis-in-an-isotropic-material-disc-subjected-to-thermal-load-and-densitywith-rigid-inclusion 


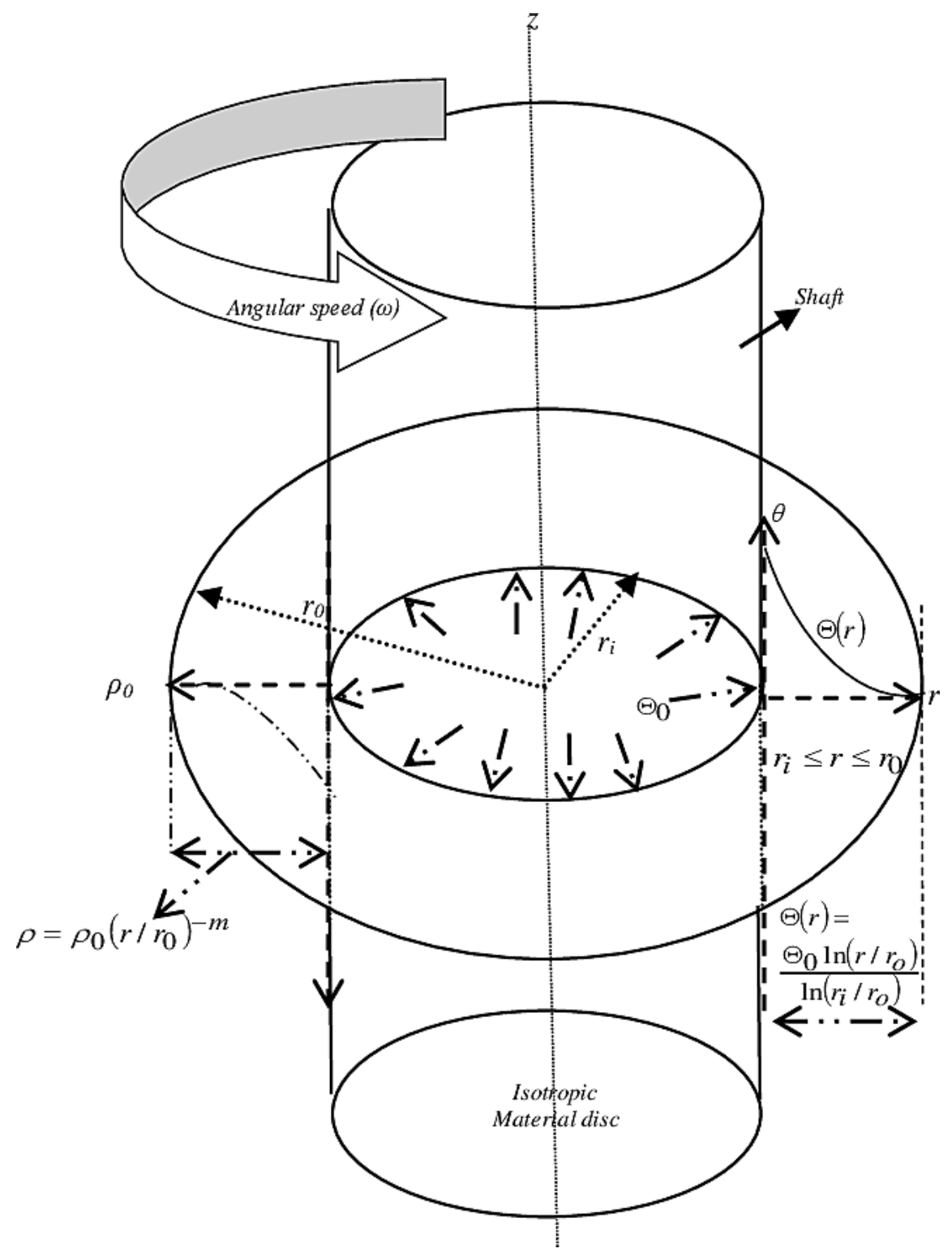



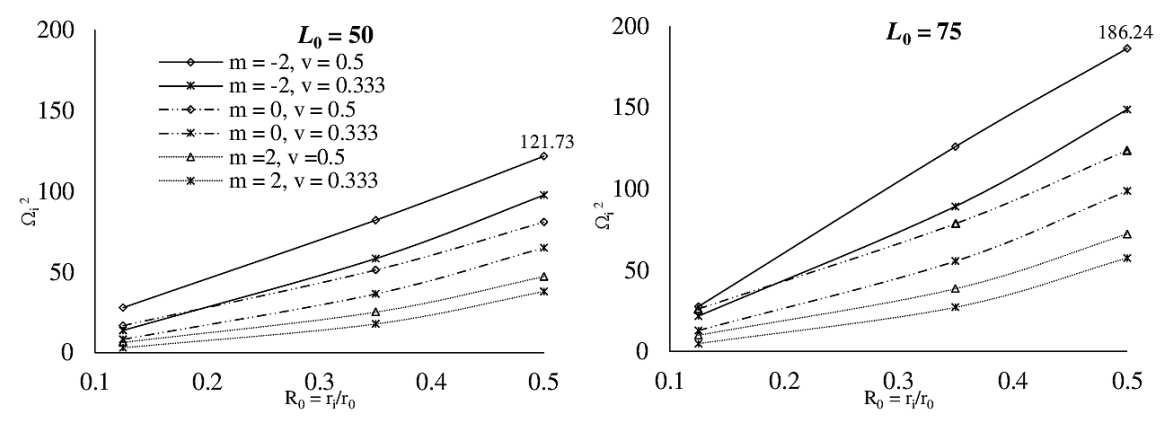

(a) Temperature $\left(\Theta_{1}=0\right)$
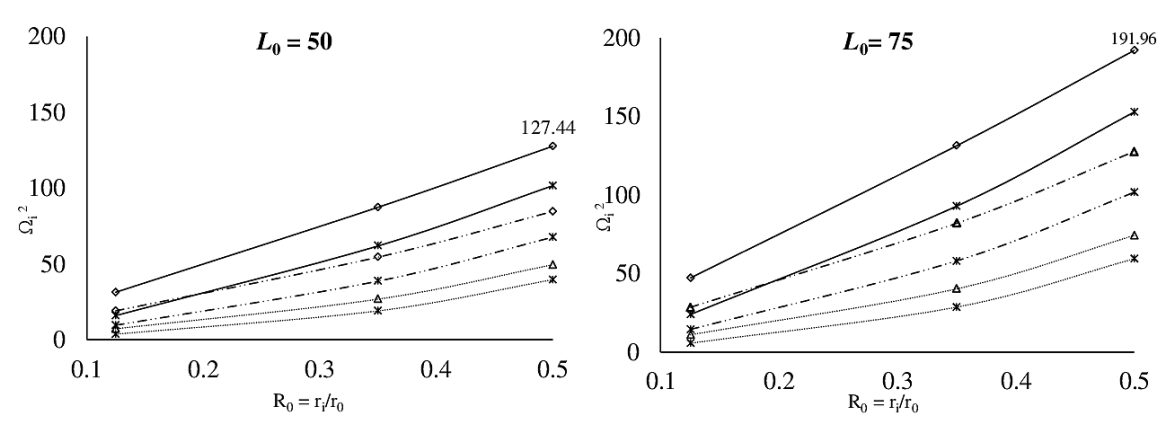

(b) Temperature $\left(\Theta_{1}=0.5\right)$
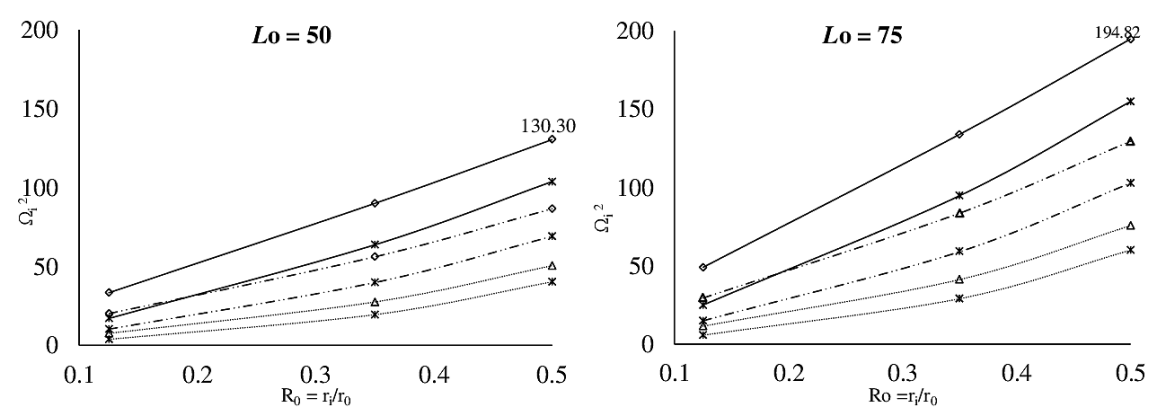

(c) Temperature $\left(\Theta_{1}=0.75\right)$ 

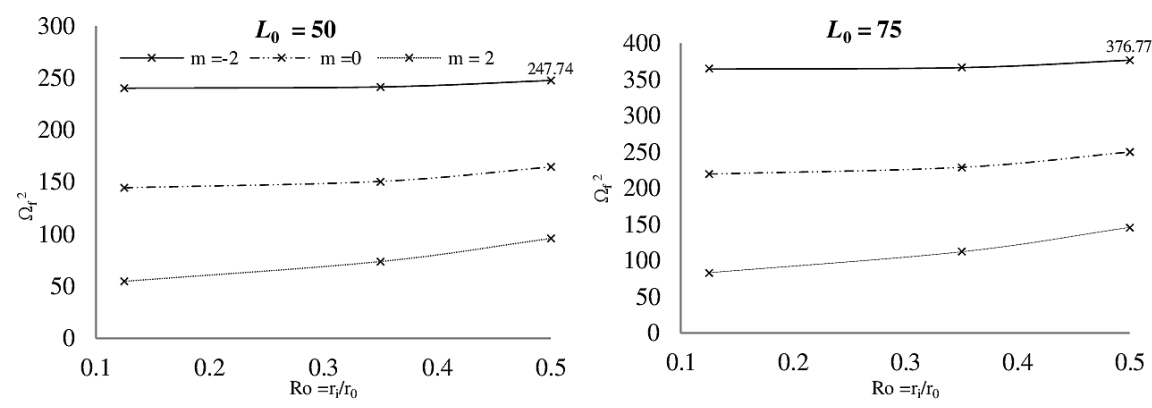

(a) Temperature $\left(\Theta_{1}=0\right)$
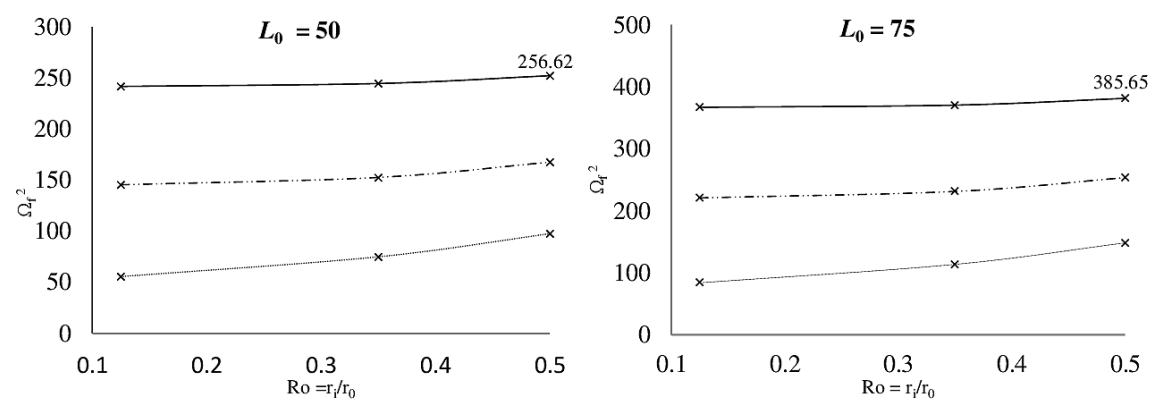

(b) Temperature $\left(\Theta_{1}=0.5\right)$
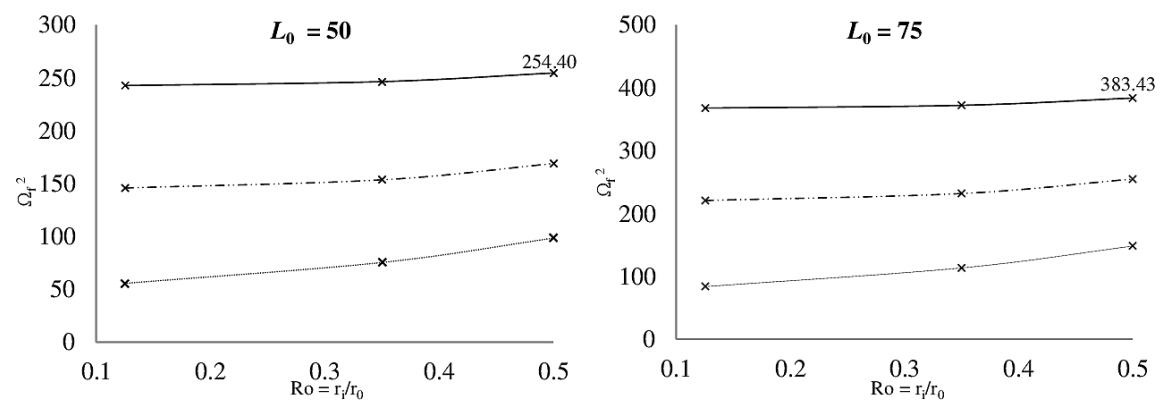

(c) Temperature $\left(\Theta_{1}=0.75\right)$ 
Meaning sigma $\mathrm{r}=\sigma_{r} ;$ sigma theta $=\sigma_{\theta}$
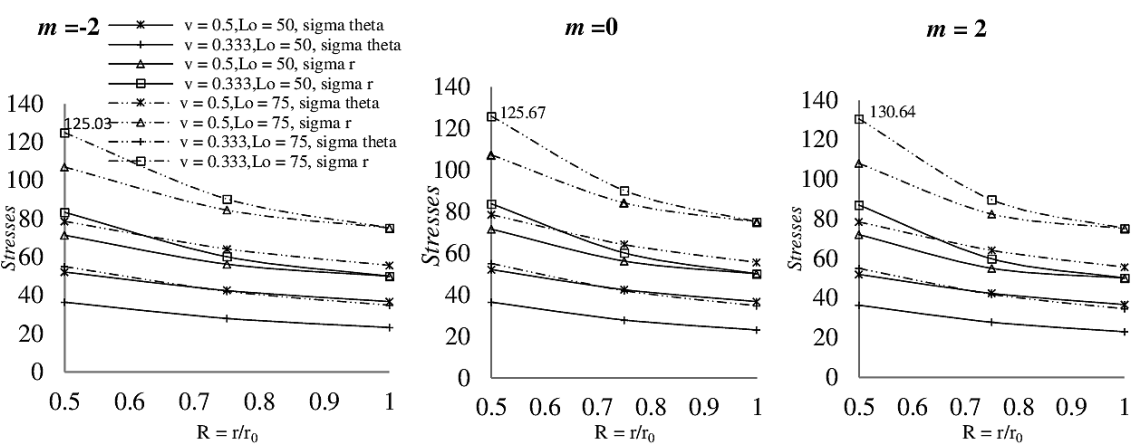

(a) Temperature $\left(\Theta_{1}=0\right)$
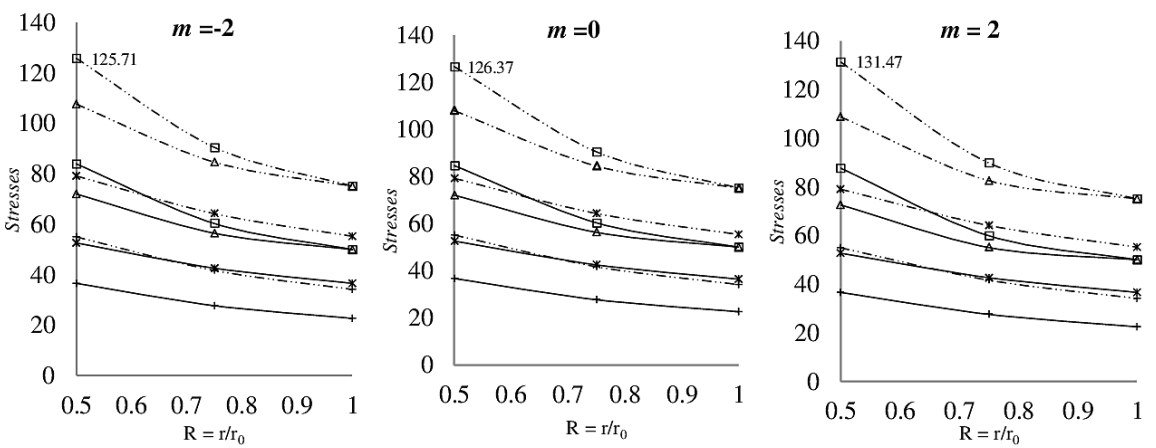

(b) Temperature $\left(\Theta_{1}=0.5\right)$
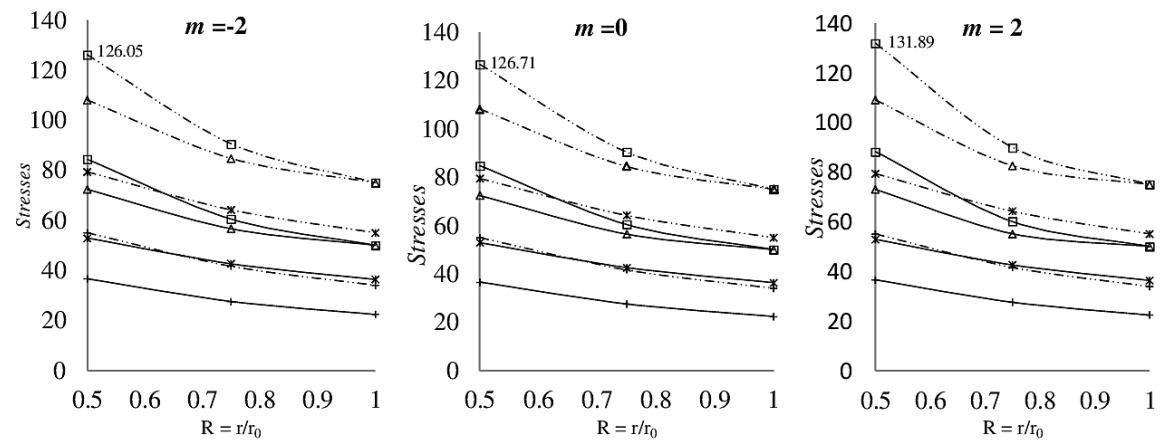

(c) Temperature $\left(\Theta_{1}=0.75\right.$ 

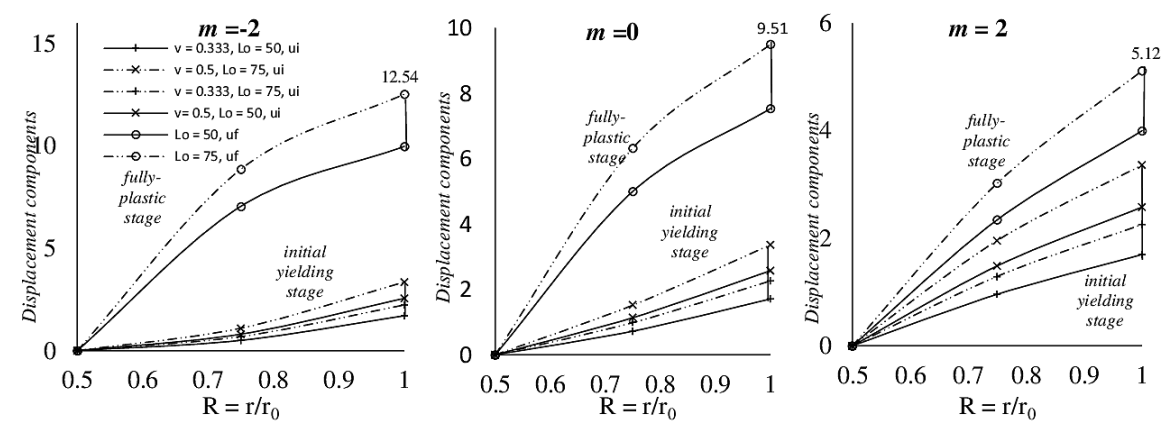

(a) Temperature $\left(\Theta_{1}=0\right)$
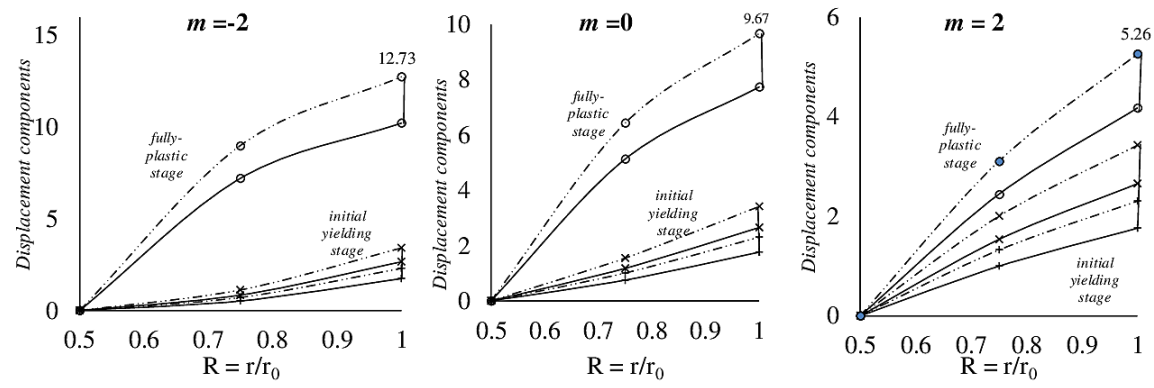

(b) Temperature $\left(\Theta_{1}=0.5\right)$
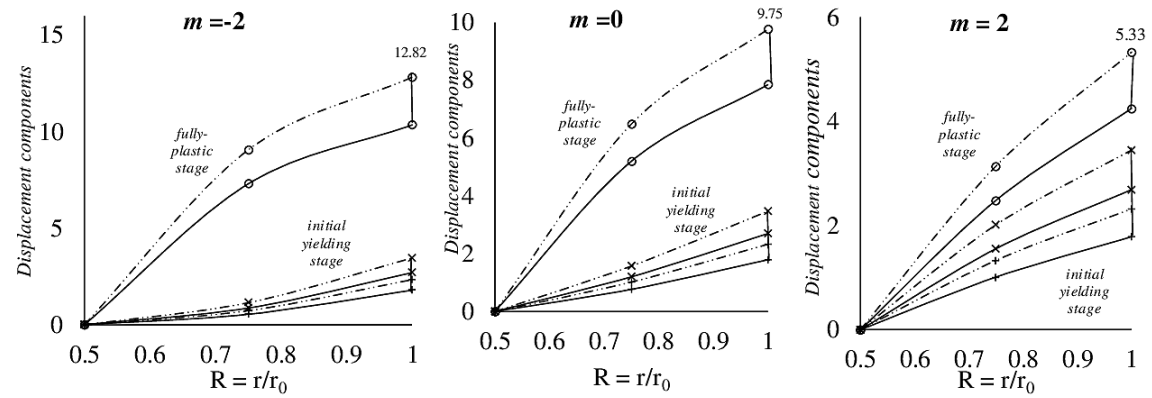

(c) Temperature $\left(\Theta_{1}=0.75\right)$ 